\title{
Review Article \\ Caudal Epidural Block: An Updated Review of Anatomy and Techniques
}

\author{
Sheng-Chin Kao and Chia-Shiang Lin \\ Department of Anesthesiology, Mackay Memorial Hospital, Taipei, Taiwan \\ Correspondence should be addressed to Chia-Shiang Lin; seancslin@gmail.com
}

Received 17 October 2016; Revised 17 December 2016; Accepted 7 February 2017; Published 26 February 2017

Academic Editor: Yasuyuki Shibata

Copyright ( 2017 Sheng-Chin Kao and Chia-Shiang Lin. This is an open access article distributed under the Creative Commons Attribution License, which permits unrestricted use, distribution, and reproduction in any medium, provided the original work is properly cited.

\begin{abstract}
Caudal epidural block is a commonly used technique for surgical anesthesia in children and chronic pain management in adults. It is performed by inserting a needle through the sacral hiatus to gain entrance into the sacral epidural space. Using conventional blind technique, the failure rate of caudal epidural block in adults is high even in experienced hands. This high failure rate could be attributed to anatomic variations that make locating sacral hiatus difficult. With the advent of fluoroscopy and ultrasound in guiding needle placement, the success rate of caudal epidural block has been markedly improved. Although fluoroscopy is still considered the gold standard when performing caudal epidural injection, ultrasonography has been demonstrated to be highly effective in accurately guiding the needle entering the caudal epidural space and produce comparative treatment outcome as fluoroscopy. Except intravascular and intrathecal injection, ultrasonography could be as effective as fluoroscopy in preventing complications during caudal epidural injection. The relevant anatomy and techniques in performing the caudal epidural block will be briefly reviewed in this article.
\end{abstract}

\section{Introduction}

The caudal epidural block involves placing a needle through the sacral hiatus to deliver medications into the epidural space. This approach to the epidural space is not only widely used for surgical anesthesia and analgesia in pediatric patients but also popular in managing a wide variety of chronic pain conditions in adults.

The caudal epidural block was first introduced as a landmark-based, blind technique. In children, the successful rate with the blind technique is above $96 \%[1,2]$. In adults, however, it was only $68-75 \%$ even in the experienced hands [3-5]. With the advent of imaging technology, fluoroscopy and ultrasonography have been increasingly used to guide caudal epidural block. In this review, we will overview recent advancement in our understanding of relevant anatomy and development of imaging guided techniques in adults.

\section{Anatomy}

The anatomic features and variations relevant to caudal epidural block were the focuses of several recent reports. A thorough knowledge of the relevant anatomy (Figures 1 and 2) may improve the success rate of caudal epidural needle placement while minimize the risks of complications.

2.1. Sacral Cornua. The sacral cornua are vestigial remnants of the inferior articular processes of the 5th sacral vertebra and presented as two bony prominences at the caudal end of sacrum. Palpating the bilateral sacral cornua is essential to locate the sacral hiatus in the conventional landmark-based technique. However, the sacral cornua are not always palpable. Defining a height of at least $3 \mathrm{~mm}$ as palpable, Sekiguchi and colleagues reported that sacral cornua were bilaterally palpable in only $19 \%$, unilaterally palpable in $25 \%$, and bilaterally impalpable in 54\% of isolated adult sacral bone [6]. Using the same definition, Aggarwal and colleagues reported that the sacral cornua were bilaterally palpable in 55\%, unilaterally palpable in $24 \%$, and bilaterally impalpable in $21 \%$ of adult sacral bone [7]. In another report, sacral cornua were not palpable bilaterally in $14.3 \%$ and palpable unilaterally in $24.5 \%$ of cadavers [8]. In a clinical report, sacral cornua were only palpable in $59 \%$ of individuals [4]. This high percentage 


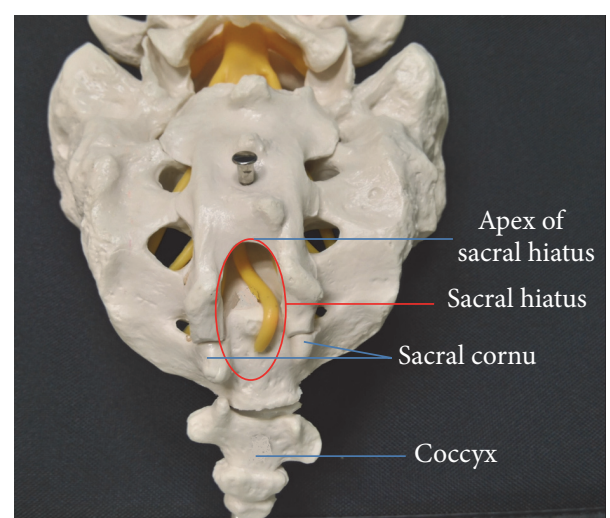

FIgURE 1: Posterior view of sacrum.

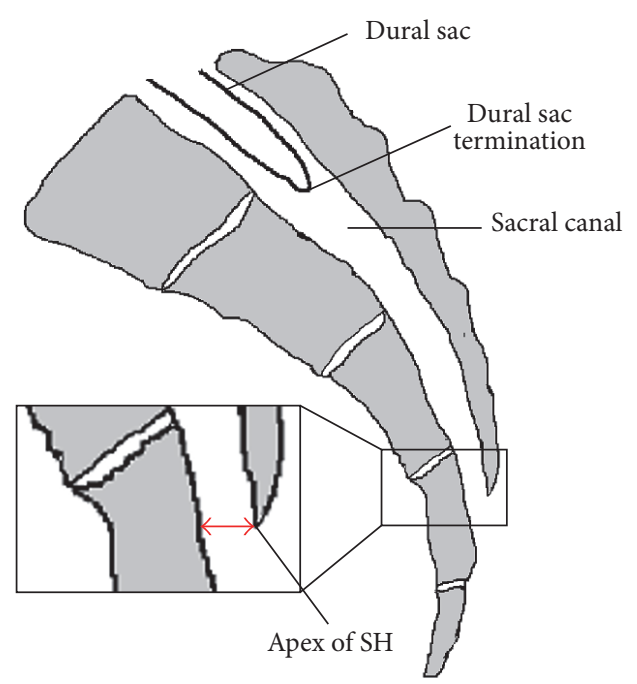

FIGURE 2: Sagittal view of sacrum. SH: sacral hiatus; red doubleended arrow: anterior-posterior diameter of sacral hiatus at its apex.

of impalpable sacral cornua may be partially accountable for the high failure rate of the blind technique.

2.2. Sacral Hiatus. The sacral hiatus, resulting from failure of fusion of lamina and spinous process of lower sacral vertebrae, is the caudal termination of the sacral canal. The sacral hiatus is bordered laterally by two sacral cornua and could be palpable as a dimple in between. Posteriorly, the sacral hiatus is covered by the skin, subcutaneous fat, and sacrococcygeal ligament (SCL). During caudal epidural block, inserting a needle into the sacral hiatus is essential to access the sacral canal. However, certain anatomic features and variations of sacral hiatus may make it difficult or impossible to insert a needle into the caudal epidural space or predispose this procedure to complications such as dual puncture.

The mean anterior-posterior (AP) diameter of sacral hiatus at its apex ranges from $4.6 \pm 2 \mathrm{~mm}$ to $6.1 \pm 2.1 \mathrm{~mm}[6,7$, 9-14] and decreased with age [14]. In clinical settings, an AP diameter of sacral hiatus at the apex of less than $3.7 \mathrm{~mm}$ was associated with difficulty in inserting a needle into the caudal epidural space by blind technique [13]. When ultrasound is used to guide needle insertion, Chen and colleagues reported that difficulty was encountered in patients with the AP diameter of sacral hiatus at apex of less than $1.6 \mathrm{~mm}$ [11]. Similar result has been reported in another study using ultrasound guidance. In that study, the average AP diameter of sacral hiatus at apex in patients with failed caudal epidural needle insertion was $1.61 \pm 0.1 \mathrm{~mm}$, significantly shorter than that $(4.7 \pm 1.7 \mathrm{~mm}, P<0.001)$ in patients with successful needle insertion [12]. The incidences of short AP diameter of sacral hiatus at its apex have been reported with different definitions. In studies using dry sacral bone, the sacral AP diameter was less than $3 \mathrm{~mm}$ in $8.77 \%$ [7] and less than $2 \mathrm{~mm}$ in $1 \%-6.25 \%$ of cases $[6,10]$. In the extreme, the sacral hiatus is completely closed, precluding inserting a needle into the caudal epidural space via the sacral hiatus. The incidence of closed sacral hiatus was $2-3 \%$ from reports studying dry human sacral bone $[6,10]$.

2.3. Location of the Apex of the Sacral Hiatus. The apex of sacral hiatus is most commonly located at the S4 level (65$68 \%$ ), followed by the S3 and S5 level (around 15\% at each level) and the S1 to S2 level in 3-5\% of cases [6, 8]. Complete agenesis of posterior wall of sacral canal (failure of fusion of sacral laminae) was noted in $1 \%$ of cases [6]. The higher the apex of sacral hiatus is located, the shorter the distance between it and the dural sac termination could be. Accidental dural puncture might occur if the needle is inserted near the apex of the sacral hiatus that is located at a high level of sacrum. On the other hand, the lower the apex of sacral hiatus is located, the shorter the length of the SCL could be. A length of the SCL of less than $17.6 \mathrm{~mm}$ was associated with difficult caudal epidural block by blind technique [13].

2.4. Dural Sac. The dural sac usually terminates between $\mathrm{S} 1$ and $S 2$ vertebra, with the majority at $S 2[8,9,15,16]$. In 1 to $5 \%$ of patients, the dural sac terminates at $\mathrm{S} 3$ or below $[15,16]$. In addition, 1 to $5 \%$ of patients with low back pain or sciatica have a sacral Tarlov cyst [15-17], a perineural cyst that communicates with the dural sac and is filled with cerebrospinal fluid (CSF). More than $40 \%$ of the sacral Tarlov cysts are located at or below the S3 level $[15,16]$. The lower the dural sac termination or the Tarlov cyst is located, the more likely dural puncture or intrathecal injection might occur during caudal epidural block.

2.5. Distance between the Dural Sac Termination and the Apex of the Sacral Hiatus. The distance between the dural sac termination and the apex of the sacral hiatus was the interest of several studies, because the risk of dural puncture is perceived to increase as this distance decreases. The average distance varies markedly from studies conducted in different ethnics. In an Indian cadaver study, the average distance was $32 \pm 12 \mathrm{~mm}$, ranging from 5.8 to $60.0 \mathrm{~mm}$ [8]. Using magnetic resonance imaging (MRI) for measurement, this distance was $60.3 \pm 13.1 \mathrm{~mm}$, ranging from 34 to $80 \mathrm{~mm}$ in a British study [9], and $44.6 \pm 11.8 \mathrm{~mm}$, ranging from 10 to $80 \mathrm{~mm}$ in a Turkish study [16]. As shown by these reports, the distance between the dural sac termination and the apex of the sacral hiatus could be as short as less than $6 \mathrm{~mm}$ in some individuals. 


\section{Techniques of Caudal Epidural Block}

3.1. Blind Caudal Epidural Block. The patient can be placed in prone or lateral decubitus position for blind caudal epidural block. A line is draw to connect the bilateral posterior superior iliac crests and used as one side of an equilateral triangle; then the location of the sacral hiatus should be approximated. By palpating the sacral cornua as 2 bony prominences, the sacral hiatus could be identified as a dimple in between. A needle is inserted at 45 degrees to the sacrum and redirected if the posterior surface of sacral bone is contacted. A subjective feeling of "give" or loss of resistance suggests piercing the SCL [18] but is associated with a miss rate up to $26 \%$ even in experienced hands [5]. The "whoosh test," performed by auscultation at the thoracolumbar region with a stethoscope while injecting $2 \mathrm{~mL}$ of air [19], has a sensitivity of $80 \%$ and a specificity of $60 \%$ in adults [20]. Palpating for subcutaneous bulging on rapid injection of $5 \mathrm{~mL}$ air or saline had a positive predictive value of $83 \%$ and a negative predictive value of $44 \%$ [4]. The inaccuracy of using blind technique for caudal epidural injection in adults, even confirmed by various tests, is clearly evident.

\subsection{Fluoroscopy-Guided Caudal Epidural Block. Because of} the inaccuracy of blind technique, some authors have recommended that caudal epidural injection is performed under fluoroscopic guidance $[3,5]$. The patient is usually placed in prone position for fluoroscopy-guided caudal epidural block. In lateral view of fluoroscopy, the sacral hiatus could be identified as an abrupt drop off at the end of S4 lamina [21]. The block needle trajectory can be visualized and navigated accordingly into the sacral canal. By injecting contrast medium under fluoroscopy, the placement of needle tip within the sacral epidural space can be verified (Figure 3), and intravascular or intrathecal needle tip placement can be detected. During caudal epidural injection, intravascular injection was reported in 3-14\% of cases by conventional fluoroscopy even after negative aspiration [3, 22, 23]. Fluoroscopy guidance has markedly improved the successful rate of caudal epidural block $[3-5,23]$ and is now considered as the gold standard in performing caudal block. However, routine use of fluoroscopy for caudal epidural block is limited by radiation exposure, cost, and special space requirement.

3.3. Ultrasound-Guided Caudal Epidural Block. The ultrasound-guided caudal block was first described by Klocke and colleagues in 2003 [24] and has, since then, gained increasing popularity. Several studies from various ethnic populations have repeatedly reported very high successful rates (96.9$100 \%)$ of ultrasound-guided caudal injection [11, 12, 2527]. The patient can be placed in prone or lateral decubitus position. Usually, a 7-13 MHz, liner transducer will suffice for most caudal epidural injection; however, a $2-5 \mathrm{MHz}$, curved transducer may be needed in obese patients. The ultrasound transducer was first placed transversely at the midline to obtain the transverse view of sacral hiatus (Figure 4). The two sacral cornua appear as two hyperechoic structures. Between the sacral cornua are two band-like hyperechoic structures;

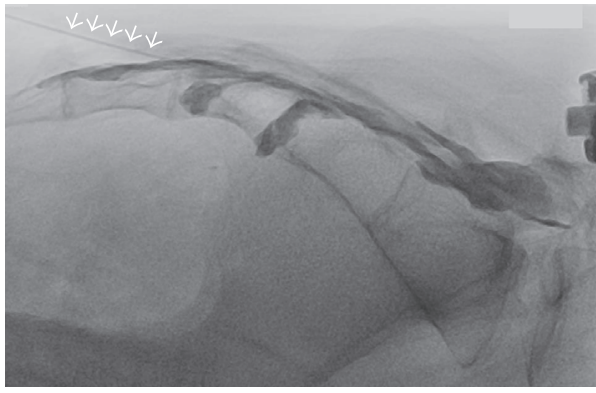

FIGURE 3: Fluoroscopy-guided caudal epidural block. Proper needle tip placement was verified by observing spread of contrast medium within the epidural space without intravascular uptake. Arrows: needle.

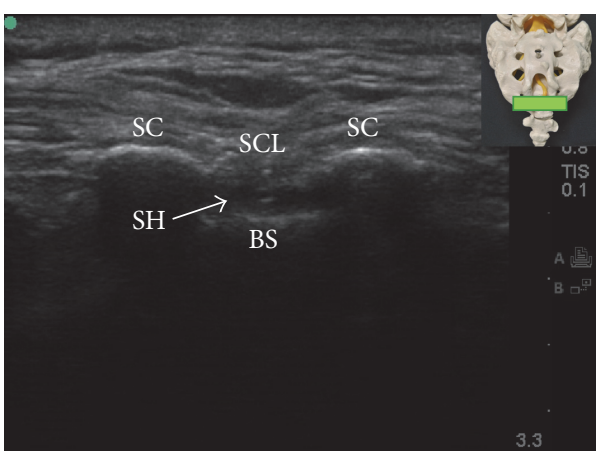

Figure 4: Transverse ultrasound view of the sacral hiatus. The inset shows the position of the ultrasound transducer. BS: base of sacrum; SC: sacral cornua; SCL: sacrococcygeal ligament; SH: sacral hiatus.

the superficial one is the SCL, and the deep one is the dorsal surface of sacral bone. The sacral hiatus was the hypoechoic region between the 2 band-like hyperechoic structures [25]. At this level, the ultrasound transducer is rotated 90 degrees to obtain the longitudinal view of sacral hiatus (Figure 5). Under longitudinal view, the block needle is inserted using the "in-plane" technique. The block needle can be visualized in real time, piercing the SCL, entering the sacral hiatus, but cannot be visualized beyond the apex of sacral hiatus. Therefore, without knowledge of dural sac termination from image study in advance, it is suggested that advancement of needle tip beyond the apex of sacral hiatus be limited to $5 \mathrm{~mm}$ to avoid dural puncture because the distance between the apex of sacral hiatus and dural sac termination can be as short as less than $6 \mathrm{~mm}[7]$.

Although ultrasonography cannot provide information regarding injectate spreading during caudal epidural injection as fluoroscopy, the presence of unidirectional flow, defined as one dominant color on color Doppler image, in the longitudinal view of sacral hiatus during injection (Figure 6) was reported to be predictive of successful caudal epidural injection $[27,28]$ and comparable treatment outcome as fluoroscopy-guided caudal epidural injection [28]. The ultrasonography could also provide information regarding the cephalad spreading of injectate during caudal epidural injection. Using a curved-array, low frequency $(2-5 \mathrm{MHz})$ 


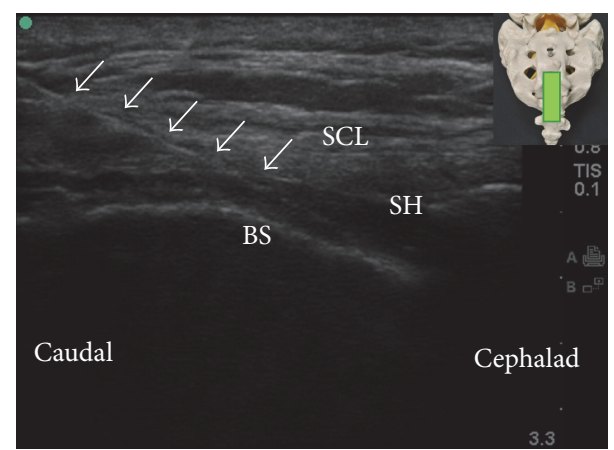

FIgURE 5: Longitudinal ultrasound view of sacral hiatus. The inset shows the position of the ultrasound transducer. BS: base of sacrum; SCL: sacrococcygeal ligament; SH: sacral hiatus; arrows: needle.

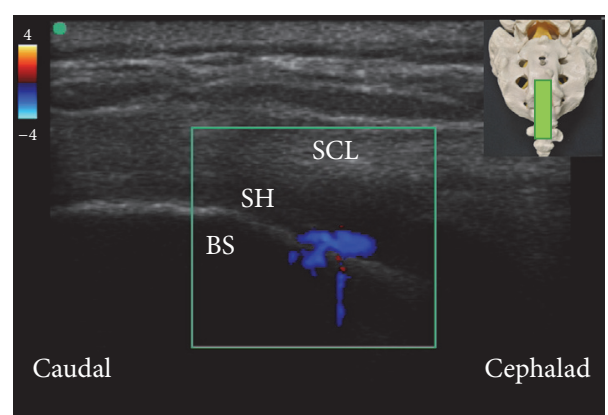

FIGURE 6: Color Doppler ultrasonography in longitudinal view of sacral hiatus. A predominantly one-color spectrum is observed in the sacral hiatus during caudal epidural injection. The inset shows the position of the ultrasound transducer. BS: base of sacrum; SCL: sacrococcygeal ligament; $\mathrm{SH}$ : sacral hiatus.

ultrasound transducer, the lumbar spinal canal could be visualized by the paramedian sagittal oblique view described by Chin and colleagues [29]. Observing color Doppler signal in the lumbar spinal canal during caudal epidural injection may indicate that the injectate has reached the lumbar epidural space (Figure 7), although this hypothesis needs to be confirmed in further studies.

While fluoroscopy with contrast medium injection is still considered the gold standard in preventing intravascular and intrathecal injection, ultrasonography could be, at least, as useful as fluoroscopy in preventing other complications during caudal epidural injection. For example, with the needle tip visualized real time going into the sacral hiatus by ultrasonography, advertently advancing the needle anteriorly into the rectum $[30,31]$ or a fetal skull in the birth canal [30] can be prevented. The practice of injecting air to verify needle tip position could be abandoned, because the injected air has been reported to cause portal vein air embolism [32] and motor weakness [33] after caudal epidural injection. In addition, ultrasound has some advantages over the fluoroscopy in guiding caudal epidural injection because it is easy to learn and radiation-free and can be virtually used in any clinical settings [25].

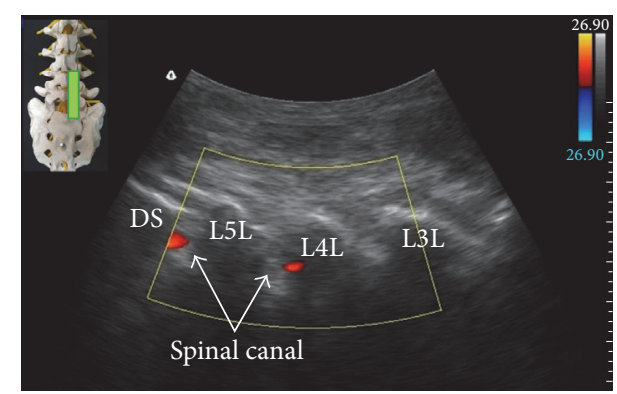

FIGURE 7: Color Doppler ultrasonography in paramedian sagittal oblique view of the sacral and lumbar spine. The observed color spectrum suggests the flow of injectate reaching L4-5 level. The inset shows the position of the ultrasound transducer. L3L: L3 lamina; L4L: L4 lamina; L5L: L5 lamina; DS: dorsal surface of sacrum.

\section{Conclusion}

There are considerable anatomic variations relevant to caudal epidural block, which may contribute to failed block by landmark-based blind technique. The advent of fluoroscopy and ultrasound has markedly improved the successful rates of caudal epidural injection. Although fluoroscopy remains the gold standard in guiding caudal epidural injection, it is not always available and radiation exposure is a concern. In addition, routine use of fluoroscopy for caudal epidural injection seems impractical in the busy operating theater and officebased clinics. Given accumulating evidence has suggested that ultrasonography is excellent in guiding caudal epidural injection with similar treatment outcome as compared with fluoroscopy-guided caudal epidural injection, ultrasound should be the preferred alternative when fluoroscopy is not available.

\section{Competing Interests}

The authors declare that there is no conflict of interests regarding the publication of this paper.

\section{References}

[1] B. Dalens and A. Hasnaoui, "Caudal anesthesia in pediatric surgery: success rate and adverse effects in 750 consecutive patients," Anesthesia and Analgesia, vol. 68, no. 2, pp. 83-89, 1989.

[2] R. M. Orme and S. J. Berg, "The 'swoosh' test-an evaluation of a modified 'whoosh' test in children," British Journal of Anaesthesia, vol. 90, pp. 62-65, 2003.

[3] D. L. Renfrew, T. E. Moore, M. H. Kathol, G. Y. El-Khoury, J. H. Lemke, and C. W. Walker, "Correct placement of epidural steroid injections: fluoroscopic guidance and contrast administration," American Journal of Neuroradiology, vol. 12, no. 5, pp. 1003-1007, 1991.

[4] M. Y. Stitz and H. M. Sommer, "Accuracy of blind versus fluoroscopically guided caudal epidural injection," Spine, vol. 24, no. 13, pp. 1371-1376, 1999.

[5] G. Barham and A. Hilton, "Caudal epidurals: the accuracy of blind needle placement and the value of a confirmatory 
epidurogram," European Spine Journal, vol. 19, no. 9, pp. 14791483, 2010.

[6] M. Sekiguchi, S. Yabuki, K. Satoh, and S. Kikuchi, "An anatomic study of the sacral hiatus: a basis for successful caudal epidural block," Clinical Journal of Pain, vol. 20, no. 1, pp. 51-54, 2004.

[7] A. Aggarwal, A. Aggarwal, Harjeet, and D. Sahni, "Morphometry of sacral hiatus and its clinical relevance in caudal epidural block," Surgical and Radiologic Anatomy, vol. 31, no. 10, pp. 793800, 2009.

[8] A. Aggarwal, H. Kaur, Y. K. Batra, A. K. Aggarwal, S. Rajeev, and D. Sahni, "Anatomic consideration of caudal epidural space: A Cadaver Study," Clinical Anatomy, vol. 22, no. 6, pp. 730-737, 2009.

[9] I. M. Crighton, B. P. Barry, and G. J. Hobbs, "A study of the anatomy of the caudal space using magnetic resonance imaging," British Journal of Anaesthesia, vol. 78, no. 4, pp. 391-395, 1997.

[10] N. Senoglu, M. Senoglu, H. Oksuz et al., "Landmarks of the sacral hiatus for caudal epidural block: an anatomical study," British Journal of Anaesthesia, vol. 95, no. 5, pp. 692-695, 2005.

[11] C. P. Chen, A. M. Wong, C.-C. Hsu et al., "Ultrasound as a screening tool for proceeding with caudal epidural injections," Archives of Physical Medicine and Rehabilitation, vol. 91, no. 3, pp. 358-363, 2010.

[12] M. Nikooseresht, M. Hashemi, S. A. Mohajerani, F. Shahandeh, and M. Agah, "Ultrasound as a screening tool for performing caudal epidural injections," Iranian Journal of Radiology, vol. 11, no. 2, Article ID e13262, 2014.

[13] Y. H. Kim, H. J. Park, S. Cho, and D. E. Moon, "Assessment of factors affecting the difficulty of caudal epidural injections in adults using ultrasound," Pain Research and Management, vol. 19, no. 5, pp. 275-279, 2014.

[14] G.-Y. Park, D. R. Kwon, and H. K. Cho, "Anatomic differences in the sacral hiatus during caudal epidural injection using ultrasound guidance," Journal of Ultrasound in Medicine, vol. 34, no. 12, pp. 2143-2148, 2015.

[15] J. Joo, J. Kim, and J. Lee, “The prevalence of anatomical variations that can cause inadvertent dural puncture when performing caudal block in Koreans: A Study Using Magnetic Resonance Imaging," Anaesthesia, vol. 65, no. 1, pp. 23-26, 2010.

[16] N. Senoglu, M. Senoglu, F. Ozkan, C. Kesilmez, B. Kizildag, and M. Celik, "The level of termination of the dural sac by MRI and its clinical relevance in caudal epidural block in adults," Surgical and Radiologic Anatomy, vol. 35, no. 7, pp. 579-584, 2013.

[17] R. D. Paulsen, G. A. Call, and F. R. Murtagh, "Prevalence and percutaneous drainage of cysts of the sacral nerve root sheath (Tarlov cysts)," American Journal of Neuroradiology, vol. 15, no. 2, pp. 293-297, 1994.

[18] R. Brull, A. J. R. MacFarlane, and V. W. S. Chan, "Spinal, epidural, and caudal anesthesia," in Miller's Anesthesia, R. D. Miller, Ed., pp. 1684-1720, Elsevier Health Sciences, 2015.

[19] M. P. N. Lewis, P. Thomas, L. F. Wilson, and R. C. Mulholland, "The "whoosh" test: a Clinical test to confirm correct needle placement in caudal epidural injections," Anaesthesia, vol. 47, no. 1, pp. 57-58, 1992.

[20] D. Eastwood, C. Williams, and I. Buchan, "Caudal epidurals: the whoosh test," Anaesthesia, vol. 53, no. 3, pp. 305-307, 1998.

[21] M. H. Landers and C. N. Aprill, "Epidural steroid injections," in Pain Procedures in Clinical Practice, T. A. Lennard, D. G. Vivian, S. D. Walkowski, and A. K. Singla, Eds., pp. 313-356, Elsevier Health Sciences, 3rd edition, 2011.
[22] W. J. Sullivan, S. E. Willick, W. Chira-Adisai et al., "Incidence of intravascular uptake in lumbar spinal injection procedures," Spine, vol. 25, no. 4, pp. 481-486, 2000.

[23] L. Manchikanti, K. A. Cash, V. Pampati, C. D. Mcmanus, and K. S. Damron, "Evaluation of fluoroscopically guided caudal epidural injections," Pain Physician, vol. 7, no. 1, pp. 81-92, 2004.

[24] R. Klocke, T. Jenkinson, and D. Glew, "Sonographically guided caudal epidural steroid injections," Journal of Ultrasound in Medicine, vol. 22, no. 11, pp. 1229-1232, 2003.

[25] C. P. C. Chen, S. F. T. Tang, T.-C. Hsu et al., "Ultrasound guidance in caudal epidural needle placement," Anesthesiology, vol. 101, no. 1, pp. 181-184, 2004.

[26] A. Blanchais, B. Le Goff, P. Guillot, J.-M. Berthelot, J. Glemarec, and Y. Maugars, "Feasibility and safety of ultrasound-guided caudal epidural glucocorticoid injections," Joint Bone Spine, vol. 77, no. 5, pp. 440-444, 2010.

[27] J. S. Yoon, K. H. Sim, S. J. Kim, W. S. Kim, S. B. Koh, and B. J. Kim, "The feasibility of color Doppler ultrasonography for caudal epidural steroid injection," Pain, vol. 118, pp. 210-214, 2005.

[28] Y. Park, J.-H. Lee, K. D. Park, J. K. Ahn, J. Park, and H. Jee, "Ultrasound-guided vs. fluoroscopy-guided caudal epidural steroid injection for the treatment of unilateral lower lumbar radicular pain: A Prospective, Randomized, Single-blind Clinical Study," American Journal of Physical Medicine and Rehabilitation, vol. 92, no. 7, pp. 575-586, 2013.

[29] K. J. Chin, M. K. Karmakar, and P. Peng, "Ultrasonography of the adult thoracic and lumbar spine for central neuraxial blockade," Anesthesiology, vol. 114, no. 6, pp. 1459-1485, 2011.

[30] C. J. Dawkins, "An analysis of the complications of extradural and caudal block," Anaesthesia, vol. 24, no. 4, pp. 554-563, 1969.

[31] V. Sathianathan and N. Dobby, "Rectal puncture complicating caudal blockade in a child with severe rectal distension," Paediatric Anaesthesia, vol. 25, no. 10, pp. 1063-1065, 2015.

[32] T. Fujikawa and S. Murai, "Portal venous gas after a caudal block," BMJ case reports, vol. 2014, 2014.

[33] M. H. Lee, C. S. Han, S. H. Lee et al., "Motor weakness after caudal epidural injection using the air-acceptance test," Korean Journal of Pain, vol. 26, no. 3, pp. 286-290, 2013. 


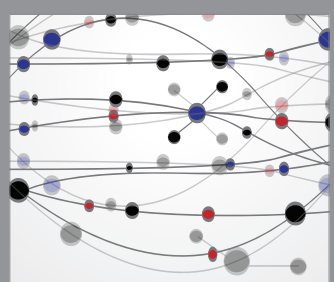

The Scientific World Journal
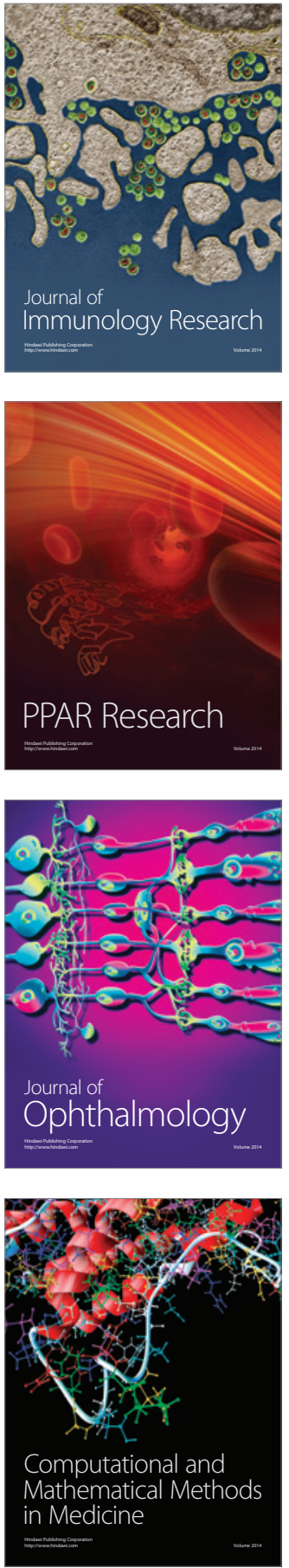

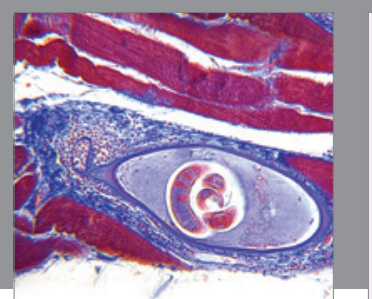

Gastroenterology Research and Practice
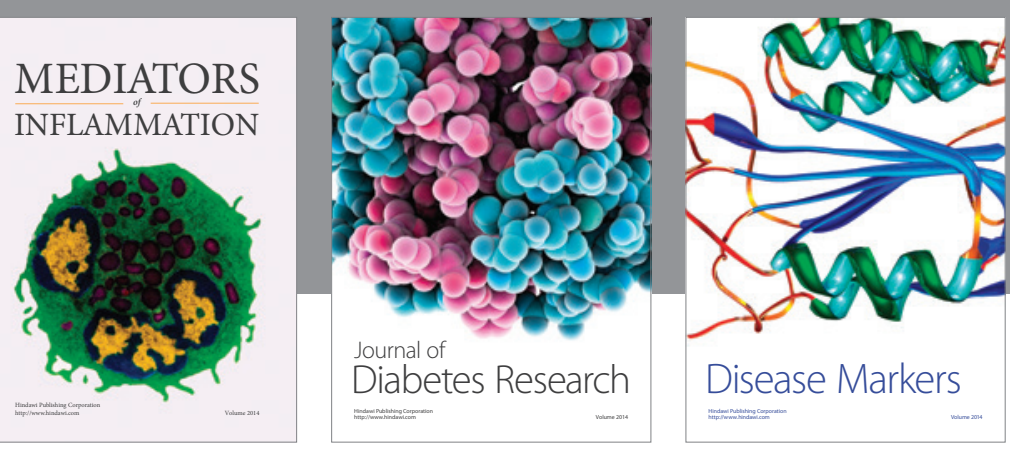

Disease Markers

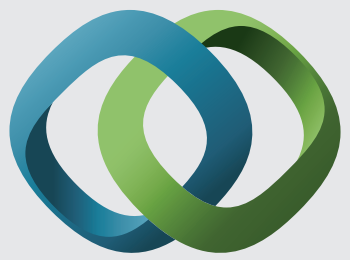

\section{Hindawi}

Submit your manuscripts at

https://www.hindawi.com
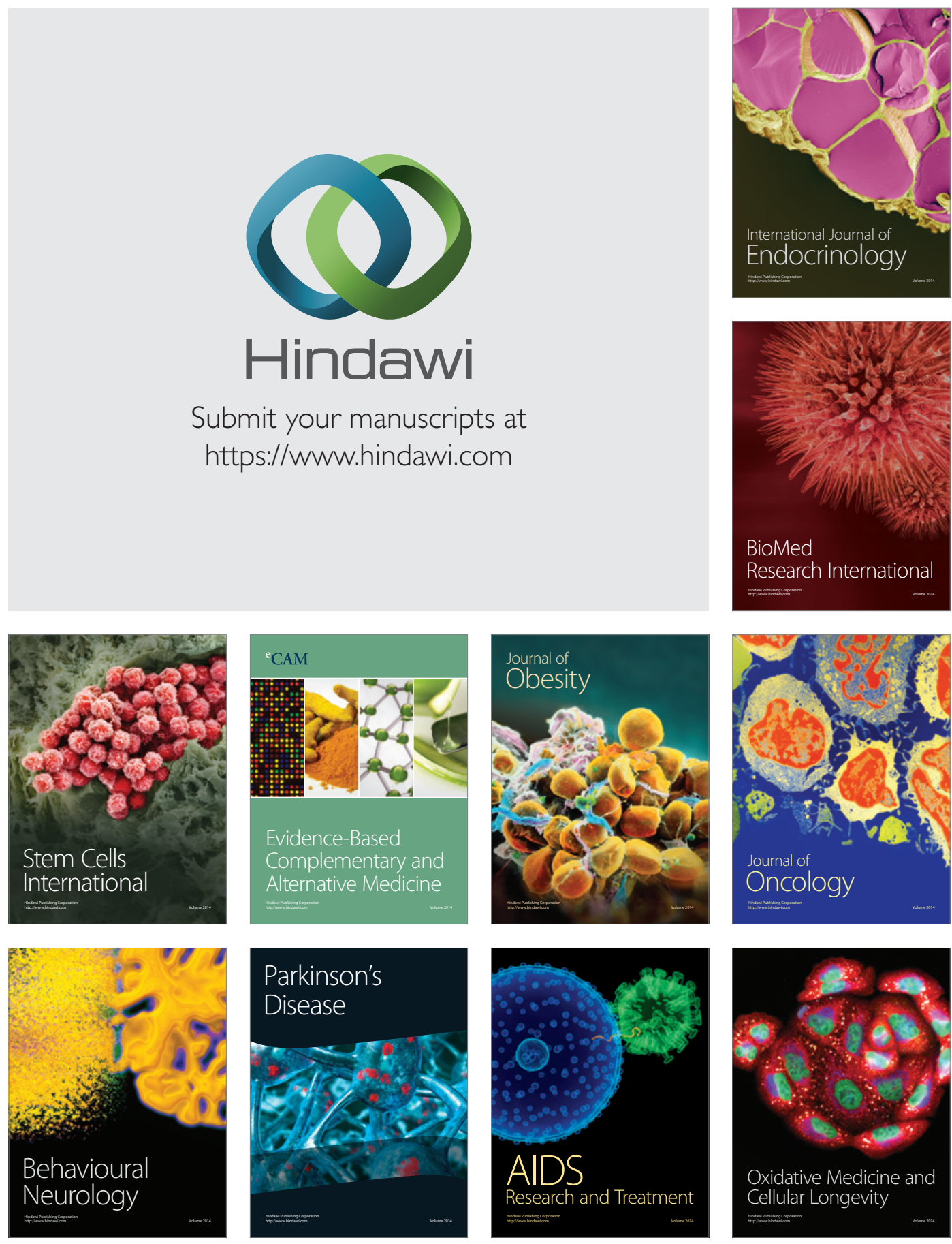